\title{
EL IMPACTO DEL GÉNERO EN LAS ELECCIONES ACADÉMICAS DE LOS ESTUDIANTES ASTURIANOS QUE FINALIZAN LA ESO
}

\author{
GENDER INFLUENCE ON ACADEMIC CHOICES OF ASTURIAN \\ STUDENTS ABOUT TO FINALIZE ESO
}

\author{
$M^{a}$ del Carmen Rodríguez Menéndez*, Susana Torío López** \\ y Carmen María Fernández García***
}

\section{RESUMEN}

En el presente artículo se estudia la persistencia de las diferencias de género que se producen en las elecciones académicas de los estudiantes asturianos que finalizan $4^{\circ}$ de la ESO. Se examinan las causas que generan esa elección diferencial, haciendo especial hincapié en el constructo "autoeficacia" como variable determinante en el proceso de elección vocacional. Se finaliza con algunas sugerencias que pueden facilitar la constitución de una orientación vocacional no sesgada por razón de género. Palabras clave: elecciones académicas, género, autoeficacia, orientación vocacional, estudio empírico, análisis estadístico.

\begin{abstract}
This paper analyzes the persistence of gender differences in academic choices of students that are about to finish the Secondary Compulsory Education. The reasons that generate that differential choice have been also examined, emphasizing the concept of "self-efficacy" as a decisive variable in the vocational process. It ends with some suggestions that may facilitate the construction of a non sexist vocational guidance.
\end{abstract}

* $M^{a}$ del Carmen Rodríguez Menéndez: Es profesora asociada del Depto. de CC. de la Educación de la Universidad de Oviedo. Sus líneas de investigación y de trabajo se relacionan con los procesos de socialización del género en el ámbito escolar y "Género y Educación”. carmenrm@uniovi.es.

** Susana Torío López: Es profesora titular y subdirectora del Dpto. de CC. de la Educación de la Universidad de Oviedo.Su línea de trabajo desarrolla y analiza las expectativas académicas y laborales de los estudiantes y su relación con la estructura familiar de procedencia. storio@uniovi.es.

*** Carmen María Fernández García: Es profesora ayudante del Depto. de CC. de la Educación de la Universidad de Oviedo. Ha participado en investigaciones relacionadas con la construcción del género en el interior de las instituciones educativas fernandezcarmen@uniovi.es.

Las tres firmantes del artículo, pertenecen al grupo de investigación ASOCED. 
Key Words: academic choices, gender, self-efficay, vocational counseling, empiric study, statistical analysis.

\section{Introducción}

La situación de las chicas en el sistema educativo ha experimentado un desarrollo notabilísimo en las últimas décadas, pues las mujeres son mayoría en todos los niveles de enseñanza. Como ejemplo de esta situación señalamos que para el curso académico 2004/2005 el porcentaje de mujeres matriculadas en la universidad española ascendía al $54 \%$ del total (M.E.C., 2006) ${ }^{1}$. Sin embargo, estos cambios no han venido acompañados de una modificación sustancial de las posiciones ocupadas en el sistema educativo. Ello es consecuencia de la persistente existencia de una segregación en la elección de las opciones académicas.

En las páginas que siguen analizamos las diferencias de género que se producen en las elecciones de las opciones académicas a las que se enfrentan los alumnos que finalizan $4^{\circ}$ de la ESO. Los datos han sido obtenidos de una investigación efectuada en el Principado de Asturias, cuyo objetivo ha sido analizar las expectativas formativas y laborales de los alumnos y alumnas que terminaban dicho curso ${ }^{2}$.

\section{Marco teórico}

Una amplia variedad de estudios han demostrado las diferencias de género que se originan en relación a las preferencias mostradas por las diversas opciones que se deben elegir al finalizar los estudios obligatorios. Se ha señalado que las chicas eligen mayoritariamente el Bachillerato de Humanidades y Ciencias Sociales; mientras que los chicos son mayoría en la opción tecnológica (Bonal, 1995; MEC, 2005; Pérez Tucho, 1993). En relación a la Formación Profesional, se indica que la presencia femenina en los ciclos formativos es menor y se limita a determinadas ramas, como son Administración y Sanidad (Solano y Frutos, 2003). Esta situación no sólo se circunscribe a nuestro país, sino que resultados similares se han hallado en estudios efectuados en el ámbito anglosajón (Betz y Schifano, 2000; Colley y Comber, 1994a, 1994b, 2003; Francis, 2002; Lightbody y Durndell, 1996; Lightbody y Siann, 1996; McKenna y Ferrero, 1991; McKinnon y Ahola-Sideway, 1995; Whitehead, 1996).

La variable "género" ejerce gran influencia en el tipo de decisiones vocacionales que los adolescentes deben acometer, por lo que se han hecho estudios para establecer los aspectos que pueden influir en la elección. En primer lugar, se ha subrayado la influencia que ejercen los procesos de socialización, pues se han interiorizado creencias y prejuicios sexistas que determinan la elección vocacional (Francis, 2002, 83). A su vez, estas creencias son reforza-

\footnotetext{
1 Los datos estadísticos elaborados por el Ministerio de Educación han sido extraídos de su página WEB: www.mec.es/educa/ccuniv/html/estadistica/Datos/DATOS0506.pdf.

2 Investigación financiada en el marco del Plan Nacional de Investigación Científica, Desarrollo e Innovación Tecnológica 2000-2003.
} 
das por las actitudes de los adultos que les rodean, e incluso el grupo de iguales puede actuar para reforzar la elección estereotipada (Alemany, 1992; Bonal, 1995; Lightbody y Durndell, 1996; McKenna y Ferrero, 1991; Vouillot et al, 2004; Whitehead, 1996).

Existen unos códigos sociales que actúan como barreras, definiendo el comportamiento individual. Este sistema de creencias y percepciones sociales influye en un aspecto de gran importancia en el estudio de las elecciones vocacionales. Nos referimos al constructo "autoeficacia", que se puede definir como "las creencias de las personas en relación a su habilidad para conducirse de modo exitoso en la ejecución de una tarea o de una conducta" (Betz y Schifano, 2000, 36. La traducción es nuestra). Además, se concluye que el comportamiento vocacional está muy influenciado por las creencias que las personas tenemos acerca de nuestra eficacia en la ejecución de determinadas tareas (Betz y Schifano, 2000; Lent y Brown, 1996; Lucas, 2000; Nevill y Schlecker, 1988; Olaz, 2003; Sullivan y Mahalik, 2000; Vouillot et al, 2004; Whiston y Bouwkamp, 2003).

Una baja percepción de autoeficacia con respecto a un domino conductual supone la evitación de esas conductas; por el contrario, unas moderadas expectativas de autoeficacia incrementan los intereses en áreas en las cuales nos sentimos eficaces y promueven la perseverancia, más allá de los obstáculos que nos podamos encontrar a lo largo de nuestra carrera académica y profesional (Hackett y Byars, 1996). Su influencia se ha mostrado tan decisiva que se han observado mayores correlaciones entre creencias de autoeficacia e intereses que entre habilidades medidas objetivamente e intereses, confirmándose el importante rol de la autoeficacia en la relación habilidad-intereses (Olaz, 2003, 363; Vouillot et al, 2004, 284).

En este contexto algunas investigaciones han demostrado la importancia de la autoeficacia percibida en la diferencias de género que acontecen en las elecciones vocacionales. Se ha concluido que las bajas expectativas de eficacia personal de hombres y mujeres para la consecución del logro en determinadas materias y ocupaciones profesionales son una barrera autoimpuesta, que supone la autoeliminación de determinados estudios y profesiones (Betz, 1992; Betz y Schifano, 2000, 35; Lent y Brown, 1996; Vouillot et al, 2004; Olaz, 366). Asimismo también se ha puesto de manifiesto que las mujeres tienen un menor desarrollo de la autoeficacia, por lo que tienen menos oportunidades de desarrollar sus habilidades potenciales (Nevill y Schlecker, 1988; Sullivan y Mahalik, 2000; Whiston y Bouwkamp, 2003).

\section{Método}

\section{Objetivos de la investigación}

Los objetivos específicos para las cuestiones que se exploran en el artículo son:

- Determinar las diferencias de género que se producen en las elecciones académicas que los estudiantes esperan efectuar al finalizar la ESO.

- Analizar las diferencias de género que se originan en la proyección manifestada por los estudiantes acerca de los estudios que desean finalizar a medio plazo.

- Estudiar las diferencias de género en las elecciones efectuadas acerca de cursos de formación ocupacional pertenecientes a distintas familias profesionales. 


\section{Selección muestral}

Se estableció una muestra representativa de los estudiantes de $4^{\circ}$ de la ESO del Principado de Asturias, usando criterios estadísticos y estratégicos. Estadísticos puesto que se respetó la proporcionalidad de la densidad escolar de cada zona geográfica de Asturias, y estratégicos porque fue necesario que determinadas zonas rurales fuesen estudiadas de forma representativa por sí misma. Además, se tuvieron en cuenta los siguientes criterios muestrales: zonas geográficas en que está dividida Asturias, titularidad de los centros, comarcas escolares en que la Consejería de Educación tiene dividida la región, relación rural y urbano, y, por último, densidad escolar. Sobre estos criterios se produjo la delimitación de cuotas y se reforzó la presencia de algunas áreas rurales, para que fueran estudiadas con verdadera representatividad.

La muestra está compuesta por 2260 sujetos, de los cuales 1077 son varones $(47.7 \%)$ y 1183 mujeres (52.3\%). El porcentaje de mujeres es ligeramente superior que el de hombres, pero esto era imposible de evitar si se quería mantener un equilibrio entre todos los criterios muestrales.

\section{Instrumento y recogida de datos}

Se construyó un cuestionario ${ }^{3}$ de 62 ítems que proporcionan una información amplia sobre las imágenes o percepciones que los alumnos han desarrollado acerca del trabajo y las distintas posibilidades formativas que tienen a su disposición. El cuestionario está compuesto de los siguientes bloques:

1. Datos sociodemográficos de la muestra: sexo, edad, estado civil del padre/ madre, nivel de estudios, profesión y situación laboral del padre/ madre, etc.

2. Impresión sobre la marcha del curso y expectativas académicas: trayectoria del curso, frecuencia de estudio, estudios que se desearía terminar y que se cree que se terminarán, decisión en caso de no superar la ESO, opción de estudios después de la ESO.

3. Expectativas laborales para el futuro: perspectiva sobre el paro y sobre el tiempo que transcurrirá antes de encontrar un empleo, aspectos más valorados del trabajo, creencia sobre el empleo futuro, aspectos que hacen necesario un trabajo, atribuciones del alumnado sobre los aspectos que influyen en la búsqueda de empleo, preferencias y rechazos profesionales.

4. Conocimiento y expectativas de formación-empleo en distintas ocupaciones: Para salvaguardar la fiabilidad y la validez de los datos recogidos la aplicación de los cuestionarios fue realizada directamente por los miembros del grupo que llevó a cabo la investigación. Además, en el momento de la recogida de los datos se indicó claramente que la información proporcionada era absolutamente confidencial. En líneas generales, se consiguió un óptimo clima de colaboración, pero si surgían dudas acerca de la fiabilidad de los datos, los cuestionarios pertinentes se desecharon.

\footnotetext{
3 Ver anexo.
} 


\section{Resultados}

Para realizar el análisis de los datos se ha empleado el programa SPSS/PC+ (versión 12.0). En la tabla $1^{4}$ recogemos las diferencias en la elección de la modalidad de Bachillerato en función del género, pues son estadísticamente significativas (Coeficiente de Contingencia $=0.296 ; \mathrm{P}=0.000$ ). Observamos una mayor presencia femenina en las modalidades de Humanidades, Ciencias Sociales y Ciencias de la Naturaleza y de la Salud; así como una equiparación intergéneros en el Bachillerato de Artes. Sin embargo, las chicas manifiestan una inclinación mucho menor que los chicos por la opción tecnológica $(9.7 \%$ frente a un $32.2 \%$ ), y se muestran menos indecisas en sus elecciones de futuro (un $8.8 \%$ de los chicos, en oposición a un $4.4 \%$ de las chicas, no sabe o no contesta al ítem).

TABLA 1: Elección de modalidades de Bachillerato en función del género.

\begin{tabular}{|c|c|c|c|c|c|c|c|}
\hline \multirow[b]{2}{*}{ Género } & \multicolumn{6}{|c|}{ Modalidades de bachillerato } & \multirow[b]{2}{*}{ Total } \\
\hline & NS/NC & Artes & $\begin{array}{c}\text { Ciencias de } \\
\text { la Naturaleza } \\
\text { y Salud }\end{array}$ & $\begin{array}{l}\text { Humanidades } \\
\text { y Ciencias } \\
\text { Sociales }\end{array}$ & Tecnología & $\begin{array}{c}\text { Varias } \\
\text { modalidades/ } \\
\text { duda }\end{array}$ & \\
\hline Hombre & $\begin{array}{l}(74) \\
8.8 \%\end{array}$ & $\begin{array}{l}(25) \\
3 \%\end{array}$ & $\begin{array}{c}(228) \\
27.1 \%\end{array}$ & $\begin{array}{c}(226) \\
26.9 \%\end{array}$ & $\begin{array}{c}(271) \\
32.2 \%\end{array}$ & $\begin{array}{l}(17) \\
2 \%\end{array}$ & $\begin{array}{l}(841) \\
100 \%\end{array}$ \\
\hline Mujer & $\begin{array}{l}(45) \\
4.4 \%\end{array}$ & $\begin{array}{c}(33) \\
3.2 \%\end{array}$ & $\begin{array}{c}(414) \\
40.7 \%\end{array}$ & $\begin{array}{c}(406) \\
39.9 \%\end{array}$ & $\begin{array}{l}(99) \\
9.7 \%\end{array}$ & $\begin{array}{l}(21) \\
2.1 \%\end{array}$ & $\begin{array}{c}(1018) \\
100 \%\end{array}$ \\
\hline Total & $\begin{array}{l}(119) \\
6.4 \%\end{array}$ & $\begin{array}{l}(58) \\
3.1 \%\end{array}$ & $\begin{array}{c}(642) \\
34.5 \%\end{array}$ & $\begin{array}{l}(632) \\
34 \%\end{array}$ & $\begin{array}{c}(370) \\
19.9 \%\end{array}$ & $\begin{array}{l}(38) \\
2 \%\end{array}$ & $\begin{array}{c}(1859) \\
100 \%\end{array}$ \\
\hline
\end{tabular}

Estas diferencias también se hacen patentes al analizar la tabla 2, que recoge las divergencias en la elección de las opciones académicas de la Formación Profesional. Dada la variedad de dichas opciones se ha optado por la agrupación en categorías. Es posible observar que en la primera fila se concentran las familias profesionales en las que la proporción de chicos electores se sitúa por encima del 90\%. En la segunda fila de la tabla se agrupan aquellas opciones que son escogidas por las chicas en porcentajes superiores al $90 \%$. La tercera, y última, fila está configurada por la única categoría profesional en las que los dos géneros aparecen representados en porcentajes similares. Siendo las diferencias estadísticamente significativas (Coeficiente de Contingencia $=0.652 ; \mathrm{P}=0.000$ ), se constata una preferencia de las chicas por las opciones vinculadas a la sanidad, imagen personal o administración en general. En oposición, los chicos se decantan por modalidades vinculadas a oficios "típicamente" masculinos (carpintería, construcción, mantenimiento de vehículos, etc.).

Los datos de la tabla 2 se complementan con los que proporciona la tabla 3 . En ella recogemos, de forma sintetizada, las respuestas a una serie de ítems elaborados para detectar

\footnotetext{
4 En las distintas tablas, la información proporcionada se refiere a los recuentos y porcentajes de cada categoría. En cada celda se recoge el recuento correspondiente para esa categoría entre paréntesis y, a continuación, se indica el porcentaje.
} 
TABLA 2: Elección de familias profesionales (FP) en función del género.

\begin{tabular}{|l|l|c|c|c|}
\hline \multicolumn{2}{|c|}{} & \multicolumn{3}{|c|}{ Género } \\
\cline { 3 - 5 } \multicolumn{1}{|l}{} & Hombre & Mujer & Total \\
\hline \multirow{4}{*}{$\begin{array}{l}\text { Modalidad } \\
\text { de FP }\end{array}$} & $\begin{array}{l}\text { Construcción, mecánica, fontanería, electricidad, } \\
\text { carpintería, mantenimiento de vehículos, } \\
\text { informática, actividades agrarias, industrias } \\
\text { alimentarias }\end{array}$ & $\begin{array}{c}(142) \\
96.5 \%\end{array}$ & $\begin{array}{c}(5) \\
3.4 \%\end{array}$ & $\begin{array}{c}(147) \\
100 \%\end{array}$ \\
\cline { 2 - 5 } & $\begin{array}{l}\text { Sanidad, imagen personal, servicios a la } \\
\text { comunidad, comercio y marketing, administración, } \\
\text { actividades deportivas, artes gráficas }\end{array}$ & $\begin{array}{c}(10) \\
8.7 \%\end{array}$ & $\begin{array}{c}(104) \\
91.2 \%\end{array}$ & $\begin{array}{c}(114) \\
100 \%\end{array}$ \\
\cline { 2 - 5 } & Hostelería y Turismo & $(8)$ & $(7)$ & $(15)$ \\
& & $53.3 \%$ & $46.7 \%$ & $100 \%$ \\
\hline
\end{tabular}

TABLA 3: Elección de oficio en función del género.

\begin{tabular}{|c|c|c|c|c|c|c|}
\hline \multirow{2}{*}{ Género } & \multicolumn{7}{|c|}{ Elección de oficio } \\
\cline { 2 - 7 } & Carpintero/a & Albañil & Cantero/a & Camarero/a & Confitero/a & $\begin{array}{c}\text { Ayudante a } \\
\text { Domicilio }\end{array}$ \\
\hline \multirow{2}{*}{ Hombre } & $(414)$ & $(333)$ & $(132)$ & $(283)$ & $(255)$ & $(125)$ \\
& $38.5 \%$ & $30.9 \%$ & $12.3 \%$ & $26.3 \%$ & $23.7 \%$ & $11.6 \%$ \\
\hline \multirow{2}{*}{ Mujer } & $(78)$ & $(34)$ & $(17)$ & $(383)$ & $(394)$ & $(299)$ \\
& $6.6 \%$ & $2.9 \%$ & $1.4 \%$ & $32.3 \%$ & $33.3 \%$ & $25.3 \%$ \\
\hline
\end{tabular}

el grado de interés que manifestaban los estudiantes por participar en cursos de formación en diferentes oficios: carpintero/a, albañil, cantero/a, camarero/a, confitero/a y ayudante a domicilio. La razón que justifica la elección de dichos oficios radica en su alta demanda laboral. Del cruce estadístico de cada uno de los oficios con la variable "género" ye que si bien chicos y chicas manifiestan un desinterés generalizado por recibir formación para dichos oficios, también se observan diferencias significativas en función del género. Se observa nuevamente un sesgo bastante acusado, de forma que los oficios de carpintero/a, albañil y cantero/a son elegidos en mayor grado por los chicos. En contraposición, las chicas son mayoría en las categorías de ayudante a domicilio, confitero/a y camarero/a; si bien con respecto a esta última ocupación deberíamos hablar de una tendencia a la equiparación de géneros. Además, las diferencias en los oficios "masculinos" son más acusadas que las

\footnotetext{
La tabla 3 es una compilación de seis cruzamientos distintos, resultado de cruzar la variable "género" con cada uno de los oficios. Todos ellos son estadísticamente significativos:

- Género/ carpintero: coeficiente de contingencia $=0.361 ; \mathrm{P}=0.000$.

- Género/ albañil: coeficiente de contingencia $=0.356 ; \mathrm{P}=0.000$.

- Género/ cantero: coeficiente de contingencia $=0.213 ; \mathrm{P}=0.000$.

- Género/ camarero: coeficiente de contingencia $=0.067 ; \mathrm{P}<0.01$.

- Género/ confitero: coeficiente de contingencia $=0.109 ; \mathrm{P}=0.000$.

- Género/ Ayudante a domicilio: coeficiente de contingencia $=0.174 ; \mathrm{P}=0.000$.
} 
mostradas para los "femeninos". Esta situación permite concluir que las chicas parecen tener muy claro que dichas opciones no forman parte de su repertorio de preferencias vocacionales.

También debemos resaltar las respuestas proporcionadas a la pregunta sobre los estudios que desean terminar. En la tabla 4 observamos que chicos y chicas reúnen porcentajes superiores al $85 \%$ con respecto al deseo de finalizar estudios de Bachillerato, FP de grado superior o universitarios. Sin embargo, un estudio más detallado de los datos permite afirmar que las chicas se sitúan casi 15 puntos por encima de los chicos en cuanto a su deseo de realizar una carrera universitaria. Al contrario, el porcentaje de chicos es mayor que el de las chicas para la categoría "Bachillerato/ FP de Grado Superior". Estos datos, que son estadísticamente significativos (Coeficiente de Contingencia $=0.149 ; \mathrm{P}=0.000$ ), se confirman con las contestaciones proporcionadas al ítem en el que se les pregunta por el tiempo que consideran que transcurrirá hasta finalizar sus estudios (tabla 5). Observamos diferencias estadísticamente significativas (Coeficiente de contingencia $=0.117 ; \mathrm{P}=0.000$ ), que mues-

TABLA 4: Estudios que desea terminar en función del género.

\begin{tabular}{|l|c|c|c|}
\hline \multirow{2}{*}{ Estudios que desea terminar } & \multicolumn{2}{|c|}{ Género } & \multirow{2}{*}{ Total } \\
\cline { 2 - 3 } & Hombre & Mujer & \\
\hline \multirow{2}{*}{ No contesta/ninguno } & $(11)$ & $(8)$ & $(19)$ \\
& $1 \%$ & $0.7 \%$ & $0.8 \%$ \\
\hline \multirow{2}{*}{ ESO } & $(47)$ & $(38)$ & $(85)$ \\
& $4.4 \%$ & $3.2 \%$ & $3.8 \%$ \\
\hline \multirow{2}{*}{ FP Grado Medio } & $(108)$ & $(88)$ & $(196)$ \\
\hline \multirow{2}{*}{ Bachillerato/ FP de Grado superior } & $10 \%$ & $7.4 \%$ & $8.7 \%$ \\
\hline \multirow{2}{*}{ Universitarios } & $(304)$ & $(220)$ & $(524)$ \\
& $28.3 \%$ & $18.6 \%$ & $23.2 \%$ \\
\hline \multirow{2}{*}{ Total } & $(606)$ & $(830)$ & $(1436)$ \\
& $56.3 \%$ & $70.1 \%$ & $63.2 \%$ \\
\hline
\end{tabular}

TABLA 5: Expectativas de tiempo de estudio en función del género.

\begin{tabular}{|l|c|c|c|c|c|}
\hline \multirow{2}{*}{ Género } & \multicolumn{3}{|c|}{ ¿Por cuánto tiempo estudiarás? } & \multirow{2}{*}{ Total } \\
\cline { 2 - 5 } & NS/NC & 2 años como máximo & 3 ó 4 años & Más de 4 años & \\
\hline \multirow{2}{*}{ Hombre } & $(31)$ & $(176)$ & $(321)$ & $(500)$ & $(1028)$ \\
& $3 \%$ & $17.1 \%$ & $31.2 \%$ & $48.6 \%$ & $100 \%$ \\
\hline \multirow{2}{*}{ Mujer } & $(31)$ & $(146)$ & $(278)$ & $(692)$ & $(1147)$ \\
& $2.7 \%$ & $12.7 \%$ & $24.2 \%$ & $60.3 \%$ & $100 \%$ \\
\hline \multirow{2}{*}{ Total } & $(62)$ & $(322)$ & $(599)$ & $(1192)$ & $(2175)$ \\
& $2.9 \%$ & $14.8 \%$ & $27.5 \%$ & $54.8 \%$ & $100 \%$ \\
\hline
\end{tabular}


tran que las chicas creen que estudiarán más tiempo. Esto coincide con sus más altas puntuaciones en el deseo de efectuar estudios universitarios (vía académica de mayor duración). Los datos coinciden con las conclusiones obtenidas por Solano y Frutos (2003), quienes indican que las chicas tienden a prolongar sus estudios formales durante más tiempo.

Aunque se observan avances significativos, como lo demuestra la mayor preferencia femenina por el Bachillerato de Ciencias de la Naturaleza y la Salud o los mayores deseos femeninos de acudir a la universidad, un análisis pormenorizado advierte que siguen existiendo opciones típicamente femeninas y otras claramente masculinas. Así, persiste la infrarrepresentación femenina en la modalidad Tecnológica del Bachillerato y en las ramas de la Formación Profesional más "masculinas". En todo caso, se debe mencionar que existe una polarización más acusada en la Formación Profesional, pues en ésta la dicotomía entre las opciones femeninas y las masculinas es muy fuerte, manteniéndose prácticamente intacta si se la compara con tiempos pasados. La situación en el Bachillerato parece más dinámica, pues hay una mayor presencia femenina en la modalidad de Ciencias de la Naturaleza y la Salud, aunque las diferencias persisten, y son muy significativas, en la opción tecnológica. Todo ello evidencia que no se ha conseguido quebrantar la asignación tradicional de los roles hombre/ mujer con respecto a las opciones vocacionales.

La situación descrita permite concluir que pese al avance que ha supuesto la mayoritaria presencia del género femenino en todos los niveles de enseñanza, así como su mejor rendimiento (Gómez Bueno et al, 2001), las chicas renuncian a las opciones académicas que gozan de un mayor prestigio y cuyo valor de cambio en el mercado laboral es superior. En consecuencia, las diferencias entre chicos y chicas se sitúan en las opciones académicas elegidas, por lo que las desigualdades entre los géneros ante el sistema educativo se han de medir, en la actualidad, por las probabilidades de paso a determinadas opciones y estudios, y no tanto por las probabilidades de éxito académico.

\section{Conclusiones}

Los resultados discutidos en el apartado anterior subrayan la necesidad de instaurar una orientación sociolaboral no sesgada por razón de género. La importancia de este hecho es mayor si cabe, si analizamos la prioridad que los estudiantes proporcionan a la orientación escolar en su proceso de toma de decisión vocacional. El 51.1\% señala que le concede bastante o mucha importancia a las orientaciones del colegio al decidir lo que estudiará o en lo que trabajará una vez finalizada la ESO. En este contexto, la orientación sociolaboral escolar debe incidir en las expectativas de autoeficacia para mejorar las elecciones vocacionales de chicos y chicas (Betz y Schifano, 2000). Desde el ámbito anglosajón ya se han elaborado programas para influir en los niveles de autoeficacia, haciendo especial incidencia en el aumento de la autoeficacia femenina. Estos programas se apoyan en cuatro recursos que forjan las creencias de autoeficacia (Betz, 1992; Hackett y Byars, 1996; Sullivan y Mahalik, 2000; Olaz, 2003):

1. Los logros de ejecución: se indica que el éxito repetido en la realización de determinadas tareas incide favorablemente en las evaluaciones positivas de autoeficacia.

2. La experiencia vicaria u observacional: la observación de modelos que se conducen de forma exitosa ayuda a los sujetos a ensayar nuevas conductas. 
3. La persuasión verbal: se señala que el refuerzo positivo de otras personas significativas aumenta la confianza del individuo sobre sus posibilidades de ejecución.

4. El estado de arousal: la existencia de elevados niveles de ansiedad en la ejecución de una tarea o habilidad pueden disminuir el rendimiento y, con ello, las creencias de autoeficacia.

El objetivo de los programas de orientación vocacional ha sido incidir en estos cuatro recursos para aumentar las expectativas de autoeficacia. Así, para desarrollar los logros de ejecución se organiza la participación de las chicas en cursos relacionados con áreas académicas que no se adecuan al estereotipo femenino. Por lo que respecta a la experiencia vicaria $u$ observacional se plantean actividades que proporcionan a las mujeres un repertorio de modelos femeninos que han tenido éxito en áreas prioritariamente masculinas (Betz, 1992). Para reducir los niveles de ansiedad en la ejecución de tareas para las que se sienten poco eficaces los programas incluyen el entrenamiento en técnicas de relajación (Betz, 1992; Betz y Schifano, 2000; Sullivan y Mahalik, 2000). Finalmente, el refuerzo verbal positivo (persuasión verbal) del orientador u orientadora es un recurso prioritario para aumentar las creencias de autoeficacia (Betz, 1992; Nevill y Schlecker, 1988).

También queremos destacar aquellas iniciativas que examinan y discuten con las mujeres las razones que explican sus bajas expectativas de autoeficacia (Betz, 1992; Lucas, 2000; McKinnon y Ahola-Sideway, 1995; Whiston y Bouwkamp, 2003). Así, se evalúan conjuntamente las barreras sociales (inexistencia de adecuados modelos de rol, prejuicios y estereotipos sociales, etc.) y personales que han generado una preferencia vocacional sesgada por razón de género. Asimismo, estos programas vocacionales también ayudan a las mujeres a analizar y valorar una mayor variedad de opciones académicas y profesionales. Para ello se trabaja en la identificación y clarificación de sus modelos de carrera y se reflexiona sobre las autoeliminaciones que efectúan.

Como complemento a estas medidas, es interesante la propuesta de Vouillot et al (2004, 287) de generar grupos de discusión de chicas con sus padres y madres. En dichos grupos se pretende evidenciar el sistema de creencias de los progenitores y analizar la influencia que han tenido en las elecciones vocacionales realizadas por sus hijas. Esta revisión y cuestionamiento de los sistemas de creencias debe alcanzar, cuando sea necesario, a los propios orientadores/ as, pues, en muchas ocasiones, no se cuestionan la representación que tienen los estudiantes de su situación y de sus proyectos de futuro (Brooks, ciatada en Vouillot et al, 2004). Por esta razón, Vouillot et al (2004) señalan la pertinencia de que los propios orientadores/ as tengan la posibilidad de interrogarse sobre sus propios esquemas mentales, en particular sobre su adhesión a los estereotipos de género.

En suma, si bien una orientación vocacional no sesgada por razón de género no debe limitarse al desarrollo de las expectativas de autoeficacia, sí debemos concluir subrayando la necesidad de desarrollar programas que favorezcan dichas expectativas, pues su logro es necesario para favorecer una elección académica y profesional no sesgada por razón de género.

\section{Referencias bibliográficas}

Alemany, C. (1992). Yo no he jugado nunca con Electro-L. (Alumnas en Enseñanza Superior Técnica). Madrid: Ministerio de Asuntos Sociales. 
Betz, N.E. (1992). "Counseling uses of career self-efficacy theory". Career Development Quarterly, Vol. 41, n' 1, 22-26.

Betz, N.E. y Schifano, R.S. (2000). "Evaluation of an interventton to increase realistic self-efficacy and interests in college women". Journal of Vocational Behaviour, Vol. 56, n 1, 35-52.

Bonal, X. (1995). Investigación-acción en educación no sexista. Una experiencia con especial referencia a la elección de estudios postobligatorios científico-técnicos en función del género. Madrid: CIDE.

Colley, A. y Comber, C. (1994a). "School subjects preferences of pupils in single sex and co-educational secondary schools". Educational Studies, Vol. 20, n 3, 379-385.

Colley, A. y Comber, C. (1994b). "Gender effects in school subject preferences: a research note". Educational Studies, Vol. 20, n 1, 13-18.

Colley, A. y Comber, C (2003). "School subject preferences: age and gender differences revisited". Educational Studies, Vol. 29, n 1, 59-67.

Francis, B. (2002). "Is the future female? The impact and implications of gender for 14-16 year olds' career choices". Journal of Education and Work, Vol. 15, $\mathrm{n}^{\circ}$ 1, 75-88.

Gómez Bueno, C.; Casares Fernández, M.; Cifuentes Martínez, C.; Carmona Bretones, A. y Fernández Palomares, F. (2001). Identidades de género y feminización del éxito académico. Madrid: Ministerio de Educación, Cultura y Deporte.

Hackett, G. y Byars, A. M. (1996). "Social cognitive theory and the career development of African American women". Career Development Quarterly, Vol. 44, n 4, 322-340.

Lent, R. W. y Brown, S. D. (1996). "Social cognitive approach to career development: an overview". Career Development Quarterly, Vol. 44, n 4, 310-321.

Lightbody, P. y Durndell, A. (1996). "Gendered career choice: is sex-stereotyping the cause or the consequence?". Educational Studies, Vol. 22, n 2, 133-146.

Lightbody, P. y Siann, G. (1996). "Motivation and attribution at secondary school: the role of gender". Educational Studies, Vol. 22, n 1, 13-25.

Lucas Mangas, S. (2000). "Estereotipos por razón de género en el autoconocimiento y autoeficacia profesional". Revista Electrónica Iberoamericana de Psicología Social: R.E.I.P.S., Vol. 1, $\mathrm{n}^{\circ} 1$.

McKenna, A. E. y Ferrero, G. W. (1991). "Ninth-grade students' attitudes toward nontraditional occupations". Career Development Quarterly, Vol. 40, n² 2, 168-181.

McKinnon, M. y Ahola-Sidaway, J. (1995). “Workin' with the boys: a north American perspective on non-traditional work initiatives for adolescent females in secondary schools". Gender and Education, Vol. 7, no 3, 327-339.

Nevill, D. D. y Schlecker, D. I. (1988). "The relation of self-efficacy and assertiveness to willingness to engage in traditional / non-traditional career activities". Psychology of Women Quarterly, 12, 91-98.

Olaz, F. O. (2003). "Autoeficacia y diferencia de géneros. Aportes a la explicación del comportamiento vocacional". Revista de Psicología General y Aplicada, Vol. 56, n 3, 359-376.

Pérez Tucho, C. (1993). La dicotomía ciencias/letras en la elección femenina de estudios universitarios. Madrid: CIDE.

Solano Lucas, J. y Frutos Balibrea, L. (2003). "Familia de origen y género como explicación de la percepción del alumnado de la nueva Formación Profesional en la región de Murcia". Tempora, 6, $175-200$.

Sullivan, K. R. y Mahalik, J. R. (2000). "Increasing career self-efficacy for women: evaluating a group intervention". Journal of Counseling \& Development, Vol. 78, $\mathrm{n}^{\circ}$ 1, 54-62. 
Vouillot, F.; Blanchard, S.; Marro, C. \& Steinbruckner, M-L. (2004). "La division sexuée de l'orientation et du travail: une question théorique et une question de pratiques". Psychologie du Travail et des Organisations, 10, 277-291.

Whiston, S. C. y Bouwkamp, J. C. (2003). "Ethical implications of career assessment with women". Journal of Career Assessment, Vol. 11, n 1, 59-75.

Whitehead, J. M. (1996). "Sex stereotypes, gender identity and subject choice at A- level". Educational Research, Vol. 38, n 2, 147-160.

Fecha de recepción: 30-05-05

Fecha de revisión: 22-05-06

Fecha de aceptación: 17-10-06 


\section{Cuestionario sobre expectativas formativas y laborales}

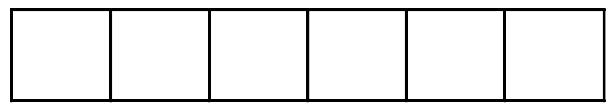

En el interior de este cuadernillo hallarás una serie de preguntas que hacen referencia a tu expectativa formativa y laboral. Para rellenar el cuestionario escribe una $(\mathbf{X})$ en el sitio que refleje tu opinión y en las demás preguntas por favor elabora una contestación lo más completa posible. Recuerda que los datos serán tratados de forma TOTALMENTE CONFIDENCIAL.

$$
* * * * *
$$

1. Sexo: Hombre $\square$ Mujer $\square$

2. Edad: $\square$ Años.

3. Lugar y país de nacimiento:

4. Si tú país de nacimiento no es España ¿Podrías decir cuántos años llevas aquí?

5. ¿Cuántos hermanos sois contándote a ti?

6. ¿Qué lugar ocupas entre tus hermanos?

7. Número de suspensos en la última evaluación

8. Número de cursos repetidos

9. Estado civil del Padre

$\begin{array}{lll}\text { Soltero } & \text { Casado } \square & \text { Separado } \\ \text { Divorciado } \square & \text { Viudo } \square & \text { Pareja de hecho } \square\end{array}$

10. Estado civil de la Madre

\begin{tabular}{|c|c|c|}
\hline Soltera & Casada & Separada \\
\hline Divorciada & Viuda & Pareja de hecho \\
\hline
\end{tabular}

11. ¿Cuáles son los estudios de más alto nivel que terminaron tus padres?

Primarios incompletos

Primarios o E.G.B

Formación Profesional de $1^{\circ}$ Grado

Bachillerato o Formación Profesional de $2^{\circ}$ Grado

Padre Madre 
Universitarios Medios

Universitarios Superiores

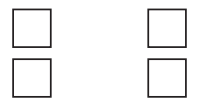

12. ¿Quién aporta el mayor número de ingresos en la economía familiar?
El padre
La madre
El padre y la madre
Hermano/a
Abuelo/a
Otras personas
¿Cuál/es?

13. ¿Cuál es la situación laboral de tu padre actualmente?

$\begin{array}{ll}\text { Parado } & \text { Activo tiempo parcial } \square \\ \text { Activo tiempo completo } \square & \text { Jubilado }\end{array}$

14. ¿Tu madre trabaja o ha trabajado fuera del hogar?

$\mathrm{Si} \square$ No $\square$

15. En caso de que la respuesta a la pregunta 14 sea afirmativa ¿Podrías decirnos, por favor, ¿cuál es su situación laboral actual?
Parada
Activa tiempo completo
Activa tiempo parcial
Jubilada

16. Cuál es la profesión principal de tu padre? Indícala con la mayor precisión:

17. Cuál es la profesión principal de tu madre Indícala con la mayor precisión:

18. ¿Cómo te va este curso?

Muy bien $\square \quad$ Bastante bien $\square \quad$ Normal $\square \quad$ Mal $\square \quad$ Muy mal

19. A la hora de buscar una razón para estudiar ¿cuál de las siguientes sería más importante para ti? ¿Y la segunda?

Me obligan mis padres

Quiero obtener buenas notas

Conseguir un buen trabajo

Para aprender

Considero una humillación tener malas notas

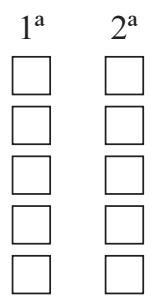


20. ¿Con qué frecuencia estudias?

Todos los días (al menos media hora)

Algún día por semana

Algún día al mes

Sólo en situaciones extraordinarias: exámenes, etc.

Nunca o casi nunca

21. Para decidir lo que vas a estudiar o en lo que vas a trabajar después de la ESO ¿qué importancia tienen para ti los siguientes aspectos?

$$
\begin{array}{cccc}
\text { Mucha } & \text { Bastante } & \text { Poca } & \text { Ninguna } \\
\text { importancia } & \text { importancia } & \text { importancia } & \text { importancia }
\end{array}
$$

La opinión de mis padres

La orientación que me dan en el colegio

Lo que me dicen mis amigos

Mis preferencias personales

Lo que vayan a hacer mis amigos

Lo que han hecho mis hermanos
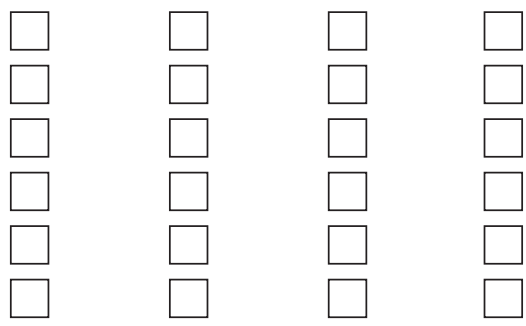

22. ¿Podrías decirme los estudios de más alto nivel que te gustaría terminar? 23. Y ¿Qué estudios crees que terminarás realmente?

Me gustaría terminar

Ninguno

E.S.O.

Formación Profesional de Grado Medio

Bachillerato o Formación Profesional de Grado Superior

Universitarios Medios

Universitarios Superiores

23. Y ¿qué estudios crees que terminarás realmente?

Creo que terminaré

Ninguno

E.S.O.

Formación Profesional de Grado Medio

Bachillerato o Formación Profesional de Grado Superior

Universitarios Medios

Universitarios Superiores

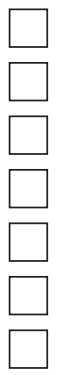


24. ¿Cómo ves tu porvenir?

$\begin{array}{llllll}\text { Ej: 1. Muy agradable } & & & \text { 4. Desagradable } \\ \text { 2. Bastante agradable } & & \text { 5. Bastante desagradable } \\ \text { 3. Agradable } & & \text { 6. Muy desagradable }\end{array}$

25. De los siguientes objetivos que uno puede marcarse en la vida puedes decirme ¿cuál es el más importante para ti? ¿Y en segundo lugar?

Luchar contra las injusticias

Casarme, tener una familia

Ganar mucho dinero

Tener un trabajo que me guste

Tener muchos amigos

Tener un trabajo bien considerado

Tener poder

Tener un trabajo estable

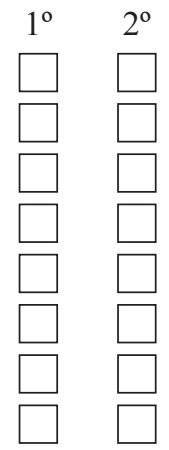

26. En relación a la definición de tu futuro profesional ¿Cómo estás?

Estoy completamente indeciso

Tengo varios proyectos pensados

Tengo un proyecto concreto

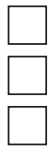


27. ¿Crees que aprobarás la ESO? $\quad \mathrm{Si} \square \quad$ No $\square$

28. En caso de superar la ESO ¿qué piensas hacer?
$\begin{array}{lll} & \text { Si } & \text { No } \\ \text { Buscaré trabajo } & \square \\ \text { Seguiré estudiando } & \square\end{array}$

29. En caso de no superar la ESO ¿qué piensas hacer?

$\begin{array}{lll} & \mathrm{Si} & \text { No } \\ \text { Buscaré trabajo } & \square\end{array}$

\section{SI PIENSAS SEGUIR ESTUDIANDO CONTESTA A LAS PREGUNTAS QUE APARECEN EN ESTE CUADRO. SI NO PASA A LA PREGUNTA 36}

30. ¿Cuál es la razón principal por la que continuarás estudiando? ¿Y la segunda más importante?

Me obligan mis padres

Quiero formarme

Conseguir un buen trabajo

Tener algo que hacer hasta que encuentre trabajo

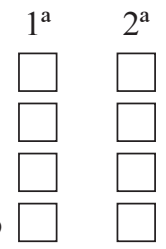

31. ¿Por cuánto tiempo estudiarás?

Dos años como máximo

Tres cuatro años

Más de cuatro años

32. Después de la ESO estudiarás:

Bachillerato

Formación Profesional

33. ¿Por cuál de las siguientes razones decides estudiar FP o Bachillerato? Señala sólo una.

Tiene más prestigio

Tiene más salida laboral

Es más fácil

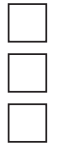

34. En caso de estudiar FP señala la modalidad 
35. En caso de estudiar Bachillerato señala la modalidad

\section{ESTA PREGUNTA SOLAMENTE LA CONTESTAS SI NO PIENSAS SEGUIR ESTUDIANDO}

36. ¿Con cuál de las siguientes situaciones te identificas más? Escoge sólo una.

a) Me gustaría seguir estudiando pero no puedo porque:

Creo que suspenderé la ESO

Debo trabajar porque en casa se necesita el dinero

En casa quiere que empiece a trabajar

Otros

b) Sólo quiero trabajar porque:

No me gusta estudiar

Para independizarme

Para cubrir mis gastos

Otros

c) No quiero ni trabajar ni estudiar

\section{A PARTIR DE ESTAS PREGUNTAS CONTESTAR TODOS}

37. ¿Piensas que estarás en paro al terminar tus estudios?

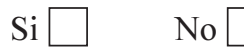

38. ¿Cuánto tiempo piensas que será preciso que transcurra para encontrar un empleo que te convenga?

Lo obtendré desde el mismo momento en que termine mis estudios

En el año siguiente de haberlos terminado

En los tres años siguientes

No lo sé

39. Pensando en el que sería un trabajo ideal para ti, ¿cuál de los siguientes aspectos valorarías más? Señala sólo uno.

Que sea seguro y estable

Que proporcione un buen sueldo

Que tenga prestigio social

40. ¿Y cuál de los siguientes te parece más interesante? Señala sólo uno.

Que te permita seguir viviendo en el mismo lugar

Que te permita tener más tiempo libre 
Que te permita decidir las horas y los días que trabajas

41. Y por último, de estos otros, ¿cuál valoras más? Señala sólo uno.

Que la tarea desarrollada sea interesante

Que ofrezca buenas oportunidades de promoción

Que sirva para ayudar a los demás

Que se corresponda con mi nivel de estudios

Que me permita realizarme

Que desarrolle mis intereses y capacidades

Que no sea monótono

Que me permita tener buenas relaciones humanas

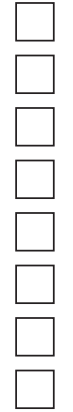

42. ¿En cuál de las siguientes situaciones te gustaría trabajar? y ¿cuál crees que será tu situación laboral en realidad?

\section{Me gustaría trabajar:}

Profesional liberal

Trabajador por cuenta ajena

Trabajador por cuenta propia

Ayudando en el negocio o en la hacienda familiar

\section{Creo que trabajaré:}

Profesional liberal

Trabajador por cuenta ajena

Trabajador por cuenta propia

Ayudando en el negocio o en la haciendafamiliar

43. ¿Cuál de los siguientes aspectos, además del salario, crees que hace necesario un trabajo? Señala sólo uno.

Permite ser alguien en la vida

Permite realizar una actividad y estar ocupado

Ofrece la oportunidad de aprender de las demás personas con las que trabajo

Se amplia el círculo de personas con las que puedo relacionarme

44. Mientras estudias la ESO ¿has realizado algún trabajo esporádicamente?
$\mathrm{Si}$
No $\square$

45. En caso afirmativo, ¿puedes especificar dónde?

En el negocio familiar

En el campo

Cuidando niños

Camarero

Otro: 
46. Ahora me gustaría que comparases cómo percibes tu situación en el futuro con la que tienen tus padres actualmente respecto a estos aspectos:

Yo tendré una formación...

La posibilidad de encontrar trabajo será...

Mi jornada de trabajo me permitirá tener un tiempo libre...

Con mi trabajo ganaré un sueldo...

La satisfacción en mi trabajo será...

La posibilidad de vivir momentos de desempleo será...

La necesidad de que los dos miembros de la pareja trabaje para llegar a fin de mes será... La posibilidad de cambiar de trabajo será... La necesidad de buscar un empleo fuera de la región será...

La posibilidad de encontrar empleo en la propia región será...

La posibilidad de encontrar un empleo para toda la vida será...

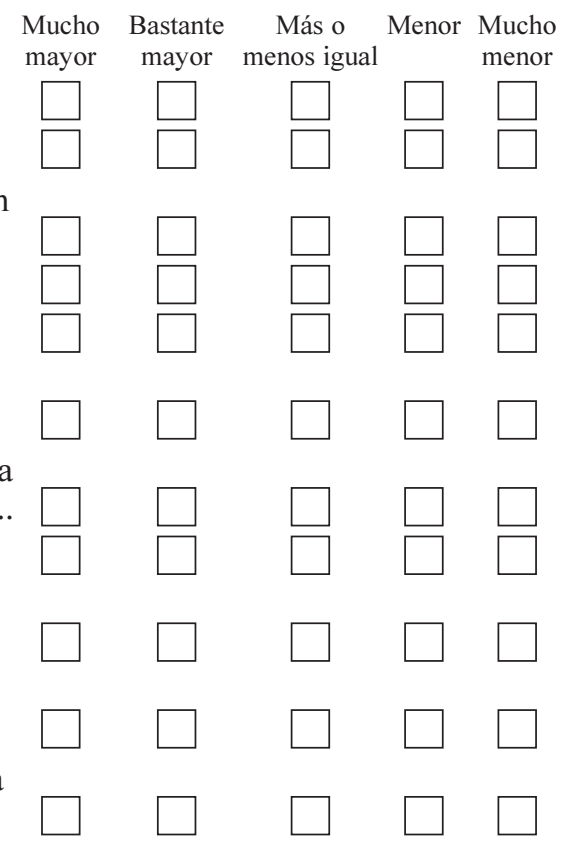

47. A la hora de encontrar un empleo, ¿qué crees que es lo que más influye?

La formación que se posea

Los enchufes

La suerte

El esfuerzo que se realiza en buscarlo

La influencia que tengan tus padres

Lo listo que sea uno
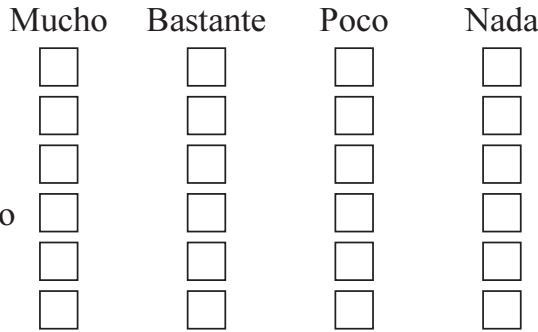

48. Escribe los tres empleos que más te gustaría desempeñar

$$
\begin{aligned}
& 1^{\circ} \\
& 2^{\circ} \\
& 3^{\circ}
\end{aligned}
$$

49. ¿Y los tres que menos?

$$
\begin{aligned}
& 1^{\circ} \\
& 2^{\circ} \\
& 3^{\circ}
\end{aligned}
$$


50. A continuación te presentamos unas profesiones. ¿Cuánto empleo crees que se oferta en cada una de ellas actualmente?

Agricultor

Jardinero

Obrero especializado de la construcción

Técnico en reparación

Comerciante, vendedor, etc.

Administrativo, procesador Informático

Empleado de una empresa de trabajo temporal

Abogado

Ingeniero

Arquitecto

Agente inmobiliario

Agente de seguros

Economista

Formador

Cuidador de ancianos

Fisoterapeuta

Peluquero

Esteticien

Camarero

Cocinero

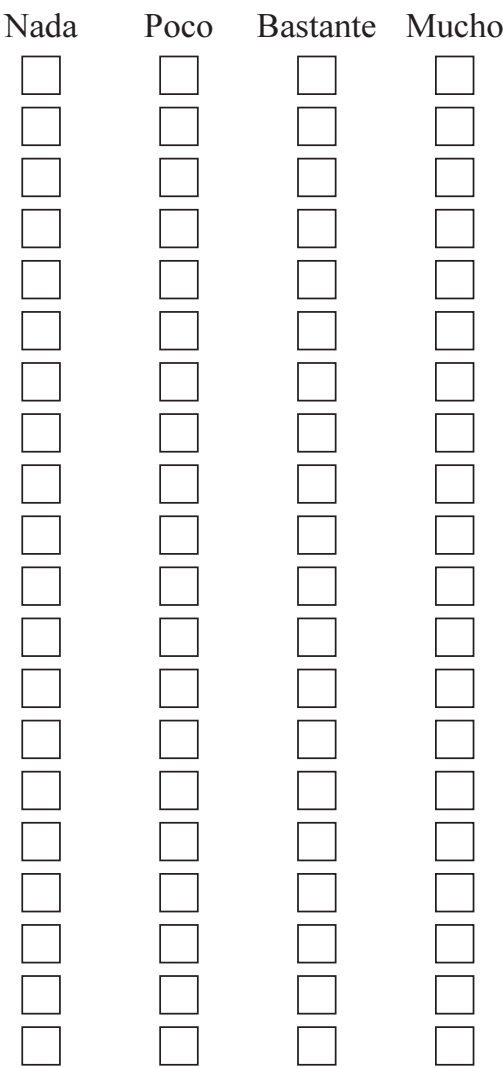

51. De las siguientes profesiones ¿elegirías alguna? En caso afirmativo señala cuál. Señalar sólo una. Y ¿cuál no elegirías? Señalar sólo una.

Sí elegiría No elegiría

Agricultor

Jardinero

Obrero especializado de la construcción

Técnico en reparación

Comerciante, vendedor, etc.

Administrativo, procesador Informático

Empleado de una empresa de trabajo temporal

Abogado

Ingeniero
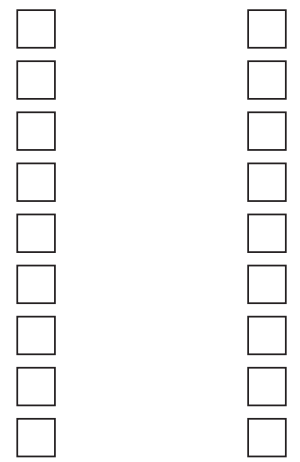
Arquitecto

Agente inmobiliario

Agente de seguros

Economista

Formador

Cuidador de ancianos

Fisoterapeuta

Peluquero

Esteticien

Camarero

Cocinero

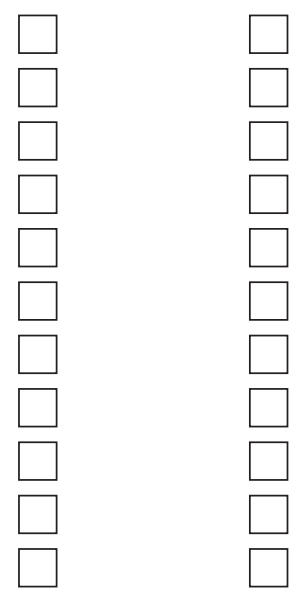

52. ¿Conoces algún lugar donde puedas aprender un oficio o profesión distinto a los centros en los que se estudia Bachillerato o Formación Profesional?

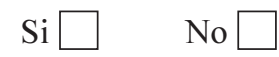

53. En caso afirmativo, ¿cuál?

54. Si te pagaran un sueldo o recibieras una beca por aprender un oficio, ¿continuarías estudiando?

$$
\mathrm{Si} \square \quad \text { No } \square
$$

55. ¿Crees que existen becas para aprender oficios?

$$
\mathrm{Si} \square \quad \text { No } \square
$$

56. En caso afirmativo, ¿cuál?

57. Después de terminar la ESO, ¿te gustaría participar en algún curso en el que te enseñaran uno de los siguientes oficios?

$\begin{array}{lll}\text { Jardinero } & \text { Si } \\ \text { Carpintero } & & \\ \text { Albañil } & & \\ \text { Cantero } & \square \\ \text { Camarero } & \square \\ \text { Confitero } & \square \\ \text { Ayuda a Domicilio } & \square\end{array}$


58. Independientemente de que los vayas a cursar o no, ¿podrías decir cómo crees que son estos oficios respecto a los siguientes aspectos?

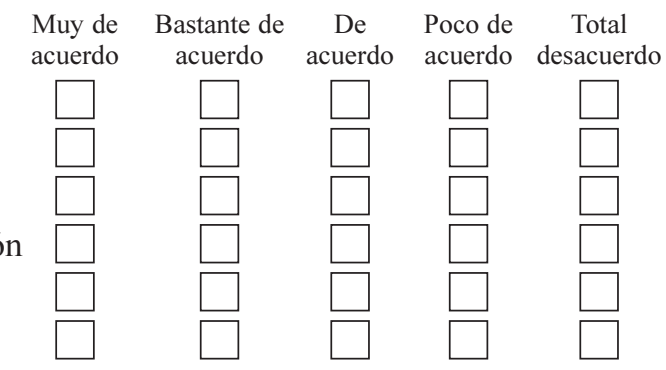

59. ¿Has oído hablar alguna vez de los siguientes programas?

Escuela Taller

Casas de Oficios

Programas de Garantía Social

Cursos del INEM

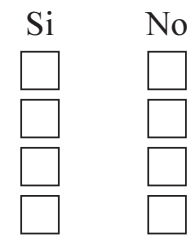

\section{EN CASO AFIRMATIVO POR FAVOR RESPONDE A LAS PREGUNTAS 60 Y 61}

60. ¿Conoces a alguien que haya participado en ellos?

$$
\text { Si } \square \quad \text { No } \square
$$

61. ¿Qué estudios crees que se necesitan para participar en estos programas?

$$
\text { Ninguno Superar Bachillerato Estudios }
$$

Escuela Taller

Casas de Oficios

Programas de Garantía Social

Cursos del INEM

la ESO o F.P. Universitarios

MUCHAS GRACIAS POR TU COLABORACIÓN 